BIO Web of Conferences 2, 04002 (2014)

DOI: $10.1051 /$ bioconf/ 20140204002

(C) Owned by the authors, published by EDP Sciences, 2014

\title{
Amino Acids in the Universe
}

\author{
J.-C. Guillemin ${ }^{1}$ \\ ${ }^{1}$ École Nationale Supérieure de Chimie de Rennes, CNRS, UMR 6226, 11 Allée de Beaulieu, CS \\ 50837, 35708 Rennes cedex 7, France. Jean-claude.guillemin@ensc-rennes.fr
}

\begin{abstract}
What is the role played by amino acids in the chemistry of the interstellar medium and the emergence of life on the early Earth? Are they really the primary key compounds as free amino acids? Is their presence or absence in the interstellar medium a significant problem? How to get an amino acid sample with an enantiomeric excess on the early Earth or ISM? How to increase the enantiomeric excess to have pure enantiomers? All these questions have received partial answers but they remain open and probably they will still be open for several more decades.
\end{abstract}

\section{Introduction}

Probably because they are essential components of proteins, the presence of amino acids is often considered as a prerequisite before any study on prebiotic compounds. In his well-known reaction, Miller showed, at the beginning of the 50', that many amino acids can be easily formed when an aqueous mixture of methane, dihydrogen and ammonia received electric discharge for several days [1]. Even if the starting mixture, chosen to simulate the atmosphere of the Primitive Earth, seems quite far from the probable composition of this atmosphere, the more recent experiments starting from dinitrogen, carbon monoxide and water also found amino acids in the products but in lower yields. Three decades after the work of Miller, Sagan observed 16 amino acids by gas chromatography after derivatization of compounds formed by acidic treatment $(6 \mathrm{~N} \mathrm{HCl})$ of tholins. These tholins were synthesized starting from methane and dinitrogen in conditions simulating electric discharges in the atmosphere of Titan [2]. On the other hand, since 1850, the famous Strecker reaction, a mixture of ammonia, hydrogen cyanide and an aldehyde in water, easily leads to the corresponding $\alpha$-aminoacetonitrile, precursor of the corresponding amino acid obtained after acidic hydrolysis (Scheme 1) [3]. 


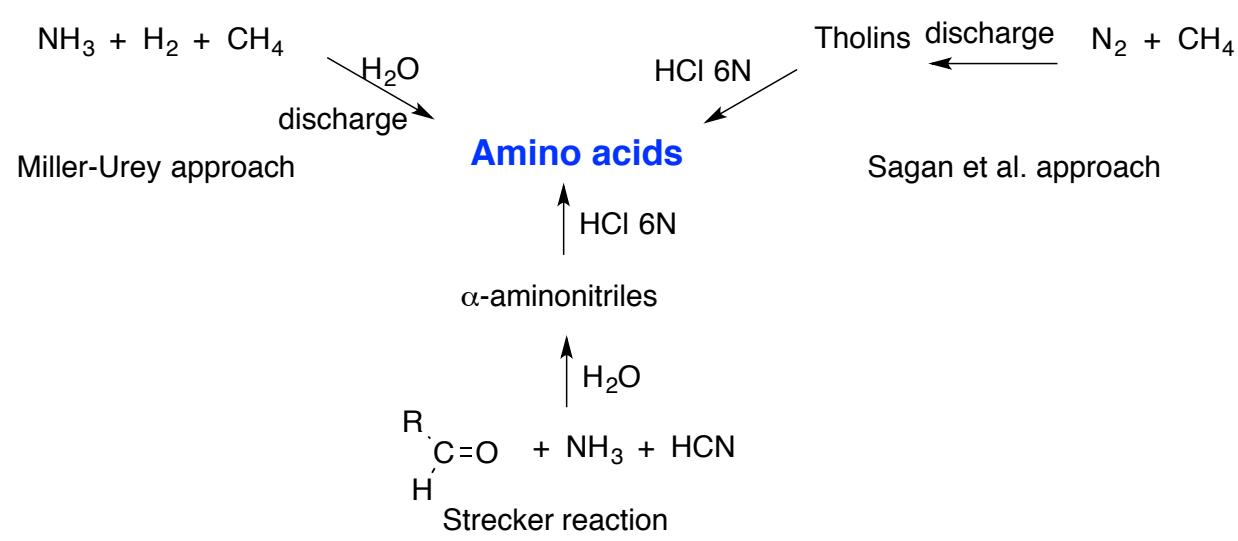

Scheme 1

So, the synthesis of amino acids is easy without sophisticated chemical conditions like a neutral atmosphere, a controlled temperature, pure reagents, ... Such compounds could be formed everywhere (interstellar medium, comets, Primitive Earth) and many studies were performed to detect them in the interstellar clouds, comaes or meteorites. Many amino acids are chiral - an enantiomer is the mirror image of the other one- (Scheme 2). Thus, studies were also devoted to generate an enantiomeric excess $(e e)$ or to increase it in the aim to give an explanation to the origin of the homochirality of living systems. In the standard process, it is also important to understand how the selection between various amino acids was performed before or during the step of peptidic couplings. We will recall here some studies showing that glycine is not the most stable isomer, how it could be formed in the ISM, and how to increase the enantiomeric excess of chiral amino acids with or without chemical transformation.<smiles>CC(N)C(=O)O</smiles>

Alanine

Scheme 2

\section{The compounds preferentially formed in the ISM and the "Minimum Energy Principle" (MEP)}

Up to now, it is quite difficult to predict which compounds are present in the ISM and we need a better knowledge of the chemistries of the ISM before to be able to do it. On the basis of compounds considered as building blocks of life on the Primitive Earth, numerous investigations have been devoted to detect in the ISM the simplest amino acid, glycine, $\mathrm{H}_{2} \mathrm{NCH}_{2} \mathrm{CO}_{2} \mathrm{H}$. But why the glycine would be present in the ISM? How could it be synthesized? Why this isomer with the $\mathrm{C}_{2} \mathrm{H}_{5} \mathrm{NO}_{2}$ formula and not another one?

When several isomers of the same formula are identified in the ISM, the " minimum energy principle » (MEP) predicts that the thermodynamically most stable compound is the most abundant [4]. The MEP is consistent with most of the observations in molecular clouds, hot cores/corinos or photodissociation regions. Thus, as examples, in the clouds where they have been detected, acetonitrile is more stable and abundant than methyl isocyanide and ketenimine, or the acetaldehyde than the vinyl alcohol (Scheme 3). 
<smiles>CNCCNCC=CC=CC=O</smiles>

Stability and abundance

Scheme 3

For non-detected compounds with the same formula than glycine, the thermodynamically most stable isomer is the carbamic acid, $\mathrm{CH}_{3} \mathrm{NHCO}_{2} \mathrm{H}$, a kinetically very unstable compound never isolated in pure form and in gas phase. The second one is the methyl carbamate $\mathrm{CH}_{3} \mathrm{OC}(\mathrm{O}) \mathrm{NH}_{2}$ and the glycine is only the third one (Scheme 4$).{ }^{4}$ Thus, glycine, with a stability about $10 \mathrm{kcal} / \mathrm{mol}$ lower than the carbamic acid and 5-6 kcal/mol lower than the methyl carbamate should be quite difficult to detect in the ISM at the present time. In fact, the microwave spectrum of the carbamic acid has never been recorded. The one of the methyl carbamate, a commercially available compound, has been analyzed [5], but up to now, this compound has never been detected in the ISM. Consequently, if the MEP can be applied, the numerous studies devoted to the detection of glycine in the ISM were really challenging. However, this very interesting concept of MEP neglects the synthesis of the compounds and it is difficult to know if that is acceptable or not.

On the other hand, attempts to detect the aminoacetonitrile, a potential precursor of glycine, and its partially hydrolyzed compound, the glycinamide, are probably more adapted to a step-by-step approach of this problem (Scheme 5). On the basis of its microwave spectrum recorded in 1990 [6], aminoacetonitrile has been detected in SgrB2, 18 years later [7]. So if the hydrolysis of aminoacetonitrile is possible in the ISM, glycine could be formed independently of the thermodynamically most stable carbamic acid isomer.<smiles>CNC(=O)OCCOC(N)=O</smiles>

Scheme 4

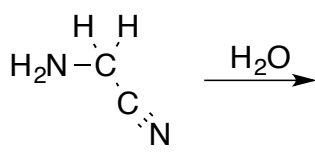<smiles>NCC(N)=O</smiles><smiles>NCC(=O)O</smiles>

Scheme 5

To illustrate the problem of the consistency between MEP and reaction pathways leading to interstellar compounds, one of our studies, devoted to the $\mathrm{C}_{4} \mathrm{H}_{5} \mathrm{~N}$ isomers, can be recalled. No member of this family has been detected up to date in the ISM. At low temperature, the thermodynamically most stable isomer is pyrrole but the flash thermolysis of various isomers never gave pyrrole (Scheme 6) [8]. An additional study should be performed by photochemistry to know if pyrrole can be formed starting from the nitriles or isonitriles isomers under irradiation. Nevertheless, other syntheses could give pyrrole without any isomer intermediate and it is difficult to know if pyrrole or crotononitrile is the most abundant in the ISM, in the case where one or several of these isomers are present in this medium. 


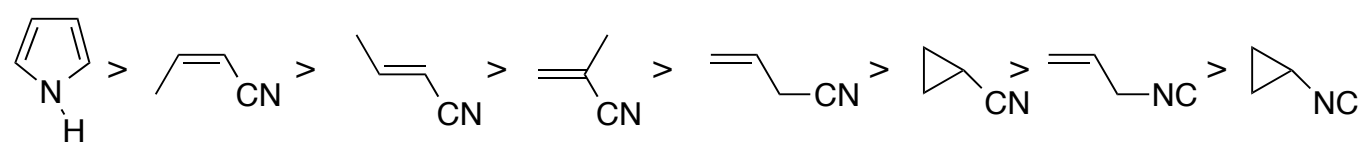

Thermodynamical stability

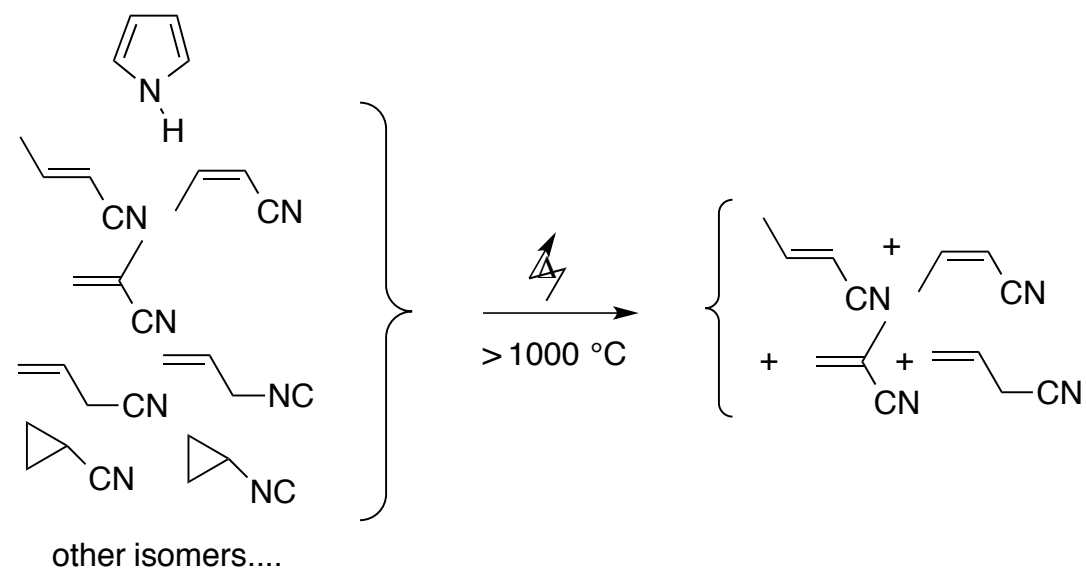

Scheme 6

Coming back to glycine, it should be noted that some recent theoretical and experimental results are in favor of glycine: 1) for the corresponding protonated species, the protonated glycine is the most stable isomer with the formula $\left(\mathrm{C}_{2} \mathrm{H}_{6} \mathrm{NO}_{2}\right)^{+}$[9], and thus, the MEP supports this glycine ion as the most abundant with this formula 2) an approach simulating the chemistry on icy grains showed that glycine can be formed via a carbamic salt as described below.

\section{Reactions on the grains at very low temperature}

In the aim to demonstrate that glycine can be formed on the grains in the interstellar medium, the thermal and photochemical behavior of methylamine in the presence of carbon dioxide has been studied. Carbon dioxide and ammonia have been observed by infrared spectroscopy on the icy grains in the ISM. The huge absorption of water renders difficult the detection of minor components but the methylamine, which has already been observed in the gas phase, could be present and maybe formed on the grains. Around 50-70 K, a temperature much higher than the one of the icy grains but possible in the hot cores, a first product was formed, the methylammonium methylcarbamate. The photolysis of this latter led to a glycine anion (Scheme 7) [10]. This approach gives a way for the formation of glycine in the interstellar medium without the preliminary formation of aminoacetonitrile and its hypothetical hydrolysis in glycine in this medium. However with a very high boiling point and a low vapor pressure at very low temperature, the presence in detectable amounts of gaseous glycine in the ISM remains still doubtful. 


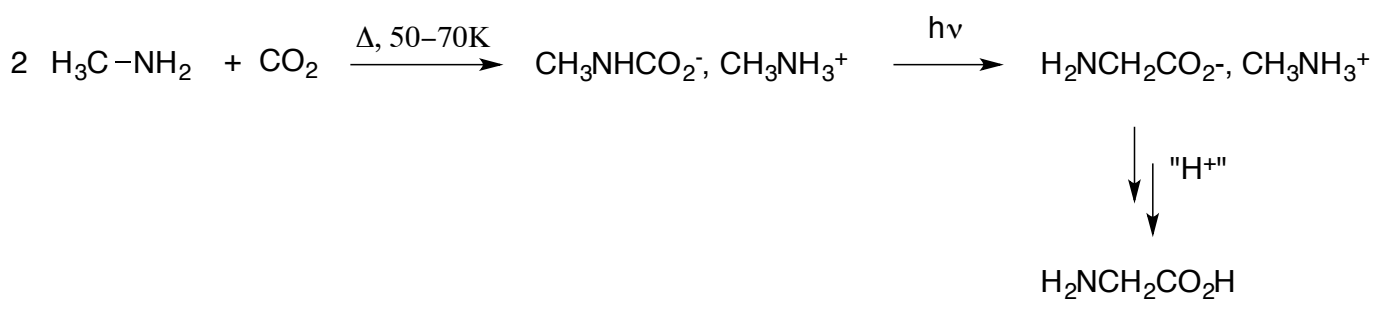

Scheme 7

\section{Amino acids in meteorites}

The meteorites contain a soluble part (MOS) and an insoluble part (MOI). Carbonaceous chondrites contain a complex suite of organic compounds that characterize these meteorites. The concentration of amino acids may reach $0.6 \mu$ mole/g meteorite $(\sim 60 \mathrm{ppm})$. Both free amino acids and acid-labile amino acid derivatives have been found in hot-water extracts of various chondrites showing, once again, the facility to form such compounds. For example, 52 amino acids have been identified in the Murchison meteorite. Thirty three of these amino acids are unknown in natural materials [11]. However, as reported above, amino acids were also found in the acidic hydrolysis of tholins coming from $\mathrm{N}_{2}-\mathrm{CH}_{4}$ mixtures subjected to electric shocks, a gaseous composition free of any oxygenated compounds. ${ }^{2}$ So, in this case, it is really clear than the oxygen atoms of amino acids are coming from water. That could be similar in part or completely for amino acids coming from the hydrolysis of meteorites but it is difficult to know which compounds are their precursors. It is important to note that such precursors could be complex molecules leading to amino acids by hydrolysis of derivatives, or, at the opposite, could come from the formation of small compounds giving the amino acids by reactions like the Strecker reaction [3] for the first step or other reactions during the aqueous washing phase. In this last case, it is difficult to conclude if such an exogeneous matter was necessary to form the amino acids on the Primitive Earth since many endogeneous organic compounds were present and able to form amino acids or precursors. The presence of a enantiomeric excess for some amino acids extracted from meteorites could support their determining role on the Primitive Earth [12].

\section{Enantiomeric excess}

Since a long time, we learned that in a reaction, without a chiral component possessing an enantiomeric excess, only racemic mixtures of chiral compounds can be formed. The works of Soai and coworkers showed that this sentence was not completely true: in very particular conditions with well-defined reagents, a stochastic behavior at the beginning of the reaction can occur and an enantiomer can be formed with an enantiomeric excess, or even in pure form [13]. Such reactions are based on a autocatalytic process where the catalyst is the product. If the first molecules of product, formed in the lack of a catalyst, possess an enantiomeric excess, this excess will determine the ee of the product at the end of the reaction. However, this particular reaction, which occurs only with an organozinc and few aldehydes, has never been extended to other reactions and particularly to the preparation of amino acids.

Up to date, the only one enantiomeric imbalance for amino acids is coming from meteorites. Nevertheless, on the Earth, mineral compounds like quartz can be found in only one enantiomeric form in some places even if, in other ones, the other enantiomer was found. Although numerous studies have been devoted to generate a first enantiomeric excess of one chiral compound, most of the studies concern samples with a more or less weak enantiomeric excess in the aim to separate an enriched sample or to increase the ee of the mixture by chemical transformations. Starting from such a small enantiomeric excess in the starting material, several physical processes allow to increase this 
ee on a sample. Sublimation [14], solubilization [15], crystallization [16] or distillation [17] are able to do it. This problem is discussed here with the sublimation.

\section{Sublimation}

When a chiral compound with an enantiomeric excess is sublimed slowly (to be not too far from an equilibrium), the first sublimate has a ee corresponding to the euatmotic (or eutectic) composition of the gas phase. The ee of this partial sublimate is thus independent on the ee of the starting material. For example, in the case of mandelic acid, this ee is of $45 \%$. If the starting material has a ee lower than $45 \%$, the partial sublimate will have a ee of $45 \%$ and the residue will have a ee lower than the starting one up to reach $0 \%[14,18]$. In the opposite case, starting from a compound with a ee higher than the one of the euatmotic composition, the ee of the partial sublimate will be still $45 \%$ but the ee of the residue will be enriched up to obtain a pure enantiomer. The ee of the gas phase at the equilibrium can be very high for some compounds: in the case of Ibuprofen, the euatmotic ee is about $90 \%$. So even starting from a sample with a small ee, the first sublimate will be $90 \%$ enriched. However, only a small sample can be extracted by this way because the residue will reach quickly $0 \%$ ee [18]. It should be noted that only a starting mixture with a ee higher than the one of the euatmotic composition can give a pure enantiomer in the residue.

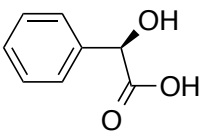

mandelic acid<smiles>CC(C)Cc1ccc([C@@H](C)C(=O)O)cc1</smiles>

ibuprofen<smiles>CC(C)C[C@H](N)C(=O)O</smiles>

leucine

Chart 1

This behavior is not easily extended to amino acids. In a first study devoted to leucine [19], we showed that the ee of the eutectic is around $45 \%$ but a very slow sublimation is necessary to obtain a sublimate with this ee (Figure 1). It is necessary to have pure leucine with a ee higher than $45 \%$ to isolate a pure enantiomer but it is not a reasonable hypothesis for the Primitive Earth. Moreover, as we will publish soon, this property of enrichment by sublimation can just be extended to some natural amino acids since many other ones decompose before to sublime. The formation of this euatmotic is also more than difficult for some of them and this property only concern pure compound, a non realistic scenario for the Primitive Earth. Up to now, postulated synergetic effects to isolate by partial sublimation the enantiomers with the same handedness and with high ees [20] have never been observed.

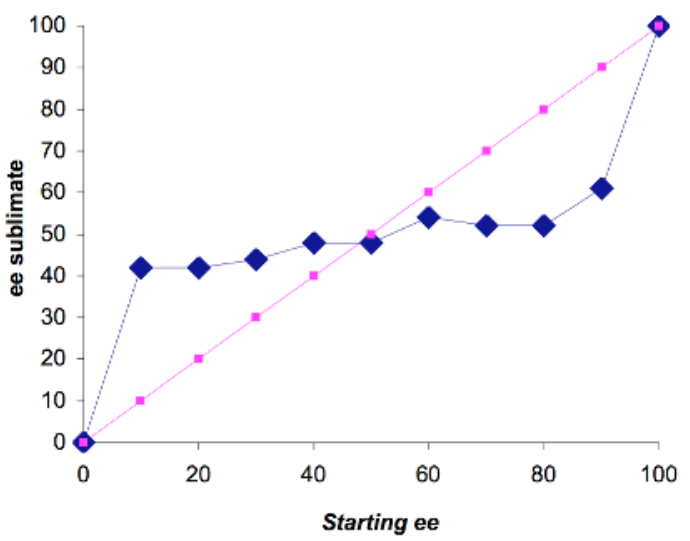

Figure 1. Slow sublimation of an enriched sample of leucine $\left(100^{\circ} \mathrm{C}, 14 \mathrm{~h}, 1-3 \%\right.$ of the starting material has been sublimed, derivatized and analyzed by chiral gas chromatography). 
On the other hand, the presence of a high boiling enantiopure amino acid mixed with more volatile racemic amino acids gives a way to separate an enriched compound. Thus, as an example, we observed that the sublimation of a mixture of $975 \mathrm{mg}$ of L-threonine and $25 \mathrm{mg}$ of leucine $(0 \%$ ee) give a partial sublimate of leucine with a $47 \%$ ee in the $D$ enantiomer. More complex mixtures give various results but, here also, a synergetic effect to extract practically pure enantiomers with the same handedness has never been demonstrated [21]. To conclude this part, the partial sublimation of enriched amino acids allows to increase the ee of one part of the starting mixture but cannot easily gives an answer to isolate pure enantiomers.

Recently some works of Viedma et al. have potentially open a new way for the enantiomeric enrichment by sublimation. The high temperature sublimation of an amino acid with an enantiomeric excess gave, after many sublimations, the same compound with a higher ee. To perform this reaction, the amino acid is introduced in a large Erlenmeyer heated at the bottom at a high temperature $\left(350^{\circ} \mathrm{C}\right)$, in conditions really far from any equilibrium of the gaseous phase. The amino acid was sublimed and condensed on the wall of the Erlenmeyer. The temperature of the wall was increasing by slow conduction of the glass wall, and the solid was thus sublimed many times up to reach the neck of the Erlenmeyer after about $15 \mathrm{~min}$. Without any reagent, an increase of the ee was observed showing that enantiomerization occurred during the sublimation. As an example, starting from a sample of valine with a $40 \% e e$, the whole sublimate has a $47 \%$ ee. This property can be also observed in the sublimation of two enriched amino acids but this approach is also, up to now, really limited in its applications [22,23].

\section{Conclusion}

Simple amino acids continue to generate many studies with connections to the origin of life, the understanding of the chemistry of the interstellar medium, comets or planetary atmospheres. But, are we sure that the free amino acids are the key building blocks of life for the first steps of the process? Are we sure that the oligomeric or polymeric compounds contained in the tholins or the meteorites are not the precursors of peptides and other prebiotic macromolecules after only a soft hydrolysis? If it is not the case, how can we imagine the formation of pure enantiomers on the Primitive Earth and the selection of the proteinogenic amino acids of the living systems among many other ones? It has been decided a long time ago that we needed the amino acids to start the story but the following steps of selections of enantiomers and selective associations in the peptidic couplings remain too difficult to be solved up to date. Why not to conclude that the easily formed amino acids are not the first primary starting materials for the long way up to the emergence of life?

\section{References}

[1] S. L. Miller, Science, 117(3046), 528-529 (1953).

[2] B. N. Khare, C. Sagan, H. Ogino, B. Nagy, C. Er, K. H. Schram, E. T. Arakawa, Icarus, 68, 17684 (1986).

[3] A. Strecker, Annalen der Chemie und Pharmazie 75, 27-45 (1850). J.-C. Guillemin, J.-M. Denis, Tetrahedron, 44, 4431-4446, (1988).

[4] M. Lattelais, F. Pauzat, Y. Ellinger, C. Ceccarelli, Astrophys. J. Lett., 696, L133 (2009).

[5] P. Groner, M . Winnewisser, I. R. Medvedev, F. C. De Lucia, E. Herbst, K. V. L. N. Sastry, 169, 28 (2008).

[6] M. Bogey, H. Dubus, J. C. Guillemin J. Mol. Spectrosc., 143, 180-182 (1990).

[7] A. Belloche, K. M. Menten, C. Comito, H. S. P. Müller, P. Schilke, J. Ott, S. Thorwirth, C. 
Hieret, Astron. \& Astrophys., 482, 179-196 (2008).

[8] M. Lattelais, Y. Ellinger, A. Matrane and J.-C. Guillemin, Phys. Chem. Chem. Phys., 12, 41654171 (2010).

[9] M. Lattelais, Y. Ellinger, B. Zanda, Internat. J. Astrobio., 6, 37-49 (2007).

[10] J.-B. Bossa, F. Duvernay, P. Theulé, F. Borget, L. d'Hendecourt, T. Chiavassa, Astron. \&Astrophys. 506, 601-608 (2009).

[11] J. R. Cronin, S. Pizzarello, Adv. Space Res. 3, 5-18 (1983).

[12] J. R. Cronin, S. Pizzarello, Science, 275, 951-955 (1997).

[13] K. Soai, I. Sato, T. Shibata, S. Komiya, M. Hayashi, Y. Matsueda, H. Imamura, T. Hayase, H. Morioka, H. Tabira, J. Yamamoto, Y. Kowata, Tetrahedron: Asymmetry 14, 185-188 (2003).

[14] J. Jacques, A. Collet, S. H. Willen, Enantiomers, Racemates and Resolutions, John Wiley \& Sons, New York (1981). Garin DL, Cooke Greco DJ, Kelley L., J. Org. Chem. 42, 1249-1251 (1977).

[15] M. Klussmann, H. Iwamura, S. P. Mathew, D. H. Wells Jr, U. Pandya, A. Armstrong, D. G. Blackmond Nature 441, 621-623 (2006). M. Klussmann, A. J. P. White, A. Armstrong, D .G. R. Blackmond, Angew Chem Int Ed 45, 7985-7989 (2006).

[16] S. Kojo, 1022-1032 (2010). S. Kojo, K. Tanaka, Chem Commun 1980-1981 (2001). S. Kojo, H. Uchino, M. Yoshimura, K. Tanaka, Chem Commun 2146-2147 (2004).

[17] B. Koppenhoefer, U. Trettin, Z Anal Chem 333, 750 (1989). T. Katagiri, C. Yoda, K. Furuhashi, K. Ueki, T. Kubota, Chem Lett 2, 115-116 (1996).

[18] A. Bellec, J.-C. Guillemin, J. Fluor. Chem., 131, 545-548 (2010).

[19] A. Bellec, A. , Guillemin, J.-C., Chem. Commun., 46, 1482-1484 (2010).

[20] S. P. Fletcher, R. B. C. Jagt, B. L. Feringa, Chem Commun, 2578-2580 (2007).

[21] A. V. Tarasevych, A. E. Sorochinsky, V. P. Kukhar, J.-C. Guillemin, Orig. Life Evol. Biosph., 43, 129-135 (2013).

[22] C. Viedma, W. L. Noorduin, J. E. Ortiz, T. de Torres, P. Cintas, Chem. Commun., 47, 671673 (2011).

[23] Cristobal Viedma, J. E. Ortiz, T. de Torres, P. Cintas Chem. Commun., 48, 3623-3625 (2012). 